Ann. Zootech., I964, 13 (2), I55-I64.

\title{
POSSIBILITÉ DE MODIFICATION DE QUELQUES CARACTÉRISTIQUES PHYSICOCHIMIQUES ET TECHNOLOGIQUES DE LA MUSCULATURE DES JEUNES BOVINS PAR UNE INJECTION D'ADRÉNALINE ANTE MORTEM
}

\author{
Y. RENOU et J. CHARPENTIER \\ avec la collaboration technique de Colette Girard et Denise GUÊnE \\ Laboratoire de Recherches sur la Viande, \\ Centre national de Recherches zootechniques, Jouy-en-Josas (Seine-et-Oise)
}

\section{SOMMAIRE}

L'influence d'une injection sous-cutanée d'adrénaline ante mortem sur quelques caractéristiques physicochimiques de la musculature a été étudiée chez des jeunes bovins castrés des races Française Frisonne Pie Noire et Normande, âgés de 16 mois.

Ce traitement a permis d'obtenir une viande présentant une coloration plus foncée, qui toutefois ne persiste pas après cuisson.

Si la viande des animaux adrénalinés était légèrement plus dure, la différence n'était pas notable.

Le point le plus important a été une amélioration de la capacité de rétention d'eau qui s'est traduite par une diminution des pertes à la cuisson.

La viande de jeunes bovins présente fréquemment une coloration insuffisante et manque de "tentie ». Ces défauts semblent liés à certaines caractéristiques biochimiques des muscles de ces animaux.

Ainsi la coloration insuffisante de ces muscles peut-être due à une trop faible teneur en pigment, ou encore à un pH musculaire trop bas (WINKLER, I939; HEDRICK et al., I959). En effet, le pH peut changer la microstructure du tissu musculaire et, par suite de modifications des conditions de réflexion de la lumière, entraîner une impression colorée différente. 
L'augmentation de la coloration peut donc être obtenue, soit par une synthèse accrue de pigment, soit par une élévation du $\mathrm{pH}$ musculaire post mortem. Cette dernière peut être provoquée par une injection d'adrénaline avant 1'abattage (HEDrick et al., I961 ; de Fremery et PoOL, I963), qui provoque une baisse du taux de glycogène musculaire, ce qui diminue la production d'acide lactique après l'abattage.

La modification du $\mathrm{pH}$ musculaire post mortem ainsi obtenue peut également avoir des répercussions sur certaines autres caractéristiques physicochimiques du tissu musculaire, en particulier sa capacité de rétention d'eau.

Les possibilités d'un tel traitement ante mortem font l'objet de la présente étude, où sont également envisagées les répercussions possibles sur certaines caractéristiques technologiques du muscle.

\section{MATÉRIEL ET MÉTHODE:}

L'expérience a porté sur i 6 animaux élevés au domaine expérimental du Pin-au-Haras par la Station de Recherches sur l'Élevage des Ruminants. Ces animaux appartenant aux races Française Frisonne Pie Noire et Normande, étaient âgés de 16 mois, avaient été castrés à 6 mois et leur poids vif moyen était de $467 \mathrm{~kg}$ ( $\pm \mathrm{I} 2)$.

Le traitement utilisé consistait en une injection sous-cutanée, au niveau du fanon, d'une solution de chlorhydrate d'adrénaline à I $\mathrm{mg}$ par ml. Cette injection, correspondant à $4 \mathrm{mg} / 100 \mathrm{~kg}$ de poids vif, était pratiquée 20 heures avant l'abattage.

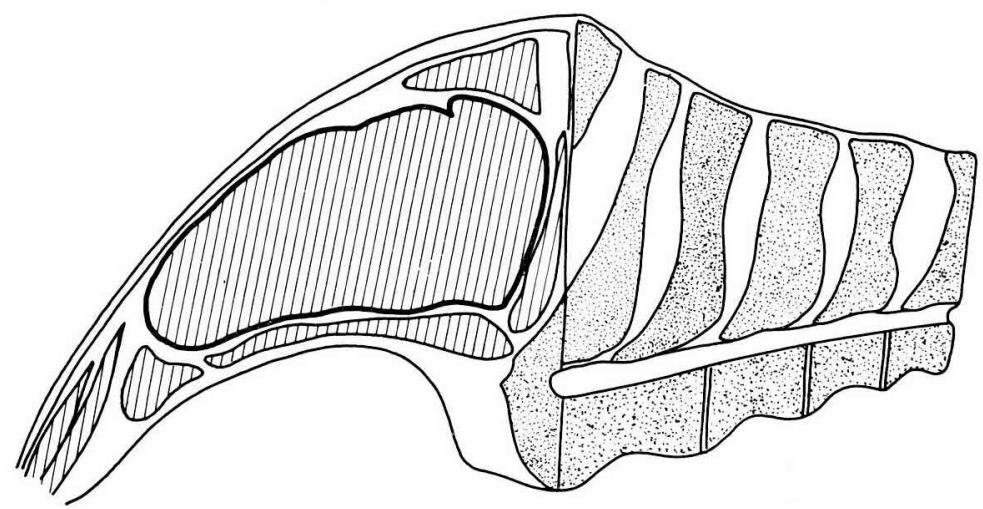

FIG. I. - Schéma d'ensemble du morceau étudié (coupe et perspective)

Les I 6 animaux étudiés ont été groupés par paires, constituées de 2 animaux aussi identiques que possible, c'est-à-dire de même race, même âge, même poids à l'abattage et même état d'engraissement. Pour cela, l'animal devant subir l'injection d'adrénaline était choisi parmi un lot homogène de 4 à 5 bêtes vivantes destinées à être abattues le même jour. L'animal témoin correspondant n'était choisi, parmi les 3 ou 4 animaux non adrénalinés du lot, qu'après l'abattage, afin de pouvoir juger plus sûrement l'état d'engraissement des carcasses. Ce dernier choix était fait par le chef de l'abattoir du C. N. R. Z., où étaient sacrifiés les animaux.

Le lendemain de l'abattage, les deux animaux étaient découpés et on prélevait sur chacun d'eux la portion d'aloyau correspondant aux deux dernières vertèbres dorsales et aux deux premières vertèbres lombaires (fig. I). 
L'ensemble de ces morceaux, conservés avec os, était placé à $+4^{\circ} \mathrm{C}$. On prélevait successivement des tranches (d'environ $20 \mathrm{~mm}$ d'épaisseur) pour la mesure des différents caractères étudiés.

Sur la portion du muscle Longissimus dorsi de ces tranches transversales, étaient effectuées les opérations suivantes:

- mesure du $\mathrm{pH}$,

- détermination du spectre de rémission (viande crue),

- détermination de la teneur en fer héminique,

- détermination du pouvoir de rétention d'eau,

- cuisson,

- détermination des pertes totales à la cuisson,

- détermination du spectre de rémission (viande cuite),

- détermination de la dureté.

Cette même sërie de mesures fut répétée, pour tous les animaux, le $2^{\mathrm{e}}$ et le $1 \mathrm{o}^{\mathrm{e}}$ jours après l'abat. tage, ainsi qu'à une $3^{\mathrm{e}}$ et une $4^{\mathrm{e}}$ dates intermédiaires, variables selon les paires d'animaux considérées. Du fait de ces prélèvements successifs, il ne nous a pas été possible de contrôler les pertes à la conservation.

\section{MÉTHODES}

\section{a) Mesure du $p H$}

Le pH était mesuré sur la tranche fraîchement coupée, à l'aide d'un pHmètre EIL $23 \mathrm{AF}$ avec une électrode de verre. La lecture était faite à o,o5 unité pH près.

Trois " sites" étaient considérés dans la tranche de Longissimus dorsi (centre, extrémité verté-

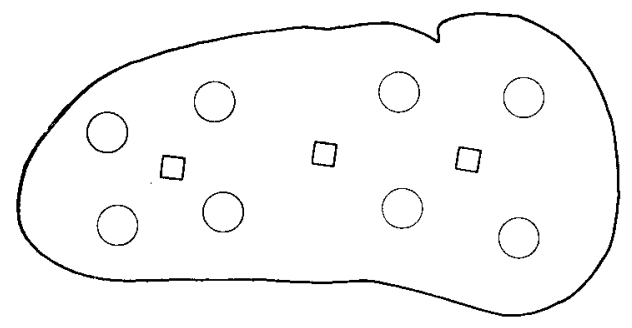

FIG 2. - Emplacements des mesures : $p H(\square)$ et dureté $(\bigcirc)$

brale, extrémité externe). (fig. 2). Chaque site faisait l'objet de quatre lectures. L'étude de la variation inter- et intrasite n'a pas justifié de distinguer ces différents sites dans les résultats. La valeur du $\mathrm{pH}$ prise en considération est donc la moyenne de ces 12 lectures.

\section{b) Spectre de rémission}

$\mathrm{b}_{1}$ ) Viande crue.

Les spectres de rémission étaient déterminés sur les tranches musculaires après une heure d'exposition à l'air. Les mesures étaient faites à l'aide d'un réflectomètre Electrosynthèse $\mathrm{SP}_{3}$ comprenant 9 filtres de bandes passantes maxima aux longueurs d'onde suivantes :

$\begin{array}{cccccccccc}\text { Filtre no } & 1 & 2 & 3 & \hat{k}_{k} & 5 & 6 & 7 & 8 & 9 \\ \lambda \text { en } \mathrm{m} \varphi & 430 & 465 & 493 & 520 & 550 & 580 & 690 & 640 & 670\end{array}$
sium.

Le tarage à 100 p. 100 de rémission de l'appareil était effectué sur un bloc de carbonate de magné-

Pour les mesures, les tranches de viande étaient appliquées sur une plaque de verre. 


\section{$\mathrm{b}_{2}$ ) Viande cuite.}

Le même processus était employé pour les mesures des spectres de rémission des tranches après cuisson. Pour cette mesure, afin d'éviter le suintement du jus, la surface des tranches était préalablement asséchée à l'aide de papier-filtre.

\section{c) Eau extraite par pression}

Pour chaque muscle, deux mesures étaient effectuées sur des échantillons de $500 \mathrm{mg}$ environ de viande hachée, par la méthode de pression (GolTfFongEA, I960).

\section{d) Cuisson}

Les tranches étaient cuites sous forme de grillades à l'aide du gril à infra-rouge d'un four électrique, à raison de 4 minutes par face.

L'estimation des pertes totales à la cuisson était calculée à partir des résultats de pesées avant et après cuisson, en pourcentage du poids initial. Les pesées étaient effectuées à $0,5 \mathrm{~g}$ près.

\section{e) Eualuation de la dureté}

Les tranches ainsi cuites étaient laissées à la température ambiante pendant environ i h $45 \mathrm{mn}$, avant qu'y soient prélevées les " carottes " destinées aux mesures de la résistance au sectionnement.

8 carottes de $\mathrm{I} 2,7 \mathrm{~mm}$ de diamètre $(\mathrm{I} / 2 \mathrm{inch})$, dont la position était répérée et constante, ont ainsi été prélevées sur chaque tranche (Fig. 2). La moyenne des 8 mesures a été prise comme indice mécanique de dureté. Ces valeurs sont exprimées en livres anglaises, telles que les donne la lecture directe sur le cadran de l'appareil « Warner-Bratzler ».

\section{f) Teneur en pigment}

Cette détermination était effectuée sur une mince tranche contiguë à celle servant aux autres déterminations.

Le pigment musculaire total (c'est-à-dire $\mathrm{Mb}+\mathrm{Hb}$ résiduelle) était déterminé par la méthode d'HORNSEY (1956) sous forme de fer héminique et exprimé en $\gamma / \mathrm{g}$ de tissu frais.

Les résultats obtenus pour ces divers caractères par les animaux des deux groupes ont été analysés conformément à la méthode d'appariement (VksSEREAU, 1948).

\section{RÉSUI'TATS - DISCUSSION}

Le tableau I donne les valeurs initiales (soit deux jours après la mort) et finales (soit dix jours après la mort) des caractéristiques de la viande, autres que les spectres de rémission qui sont représentés sur les graphiques I à 4 .

\section{I) Teneur en pigment}

Comme le montre le tableau i, la teneur en pigment est sensiblement identique pour les deux groupes d'animaux, les muscles des animaux témoins étant toutefois légèrement plus riches. 

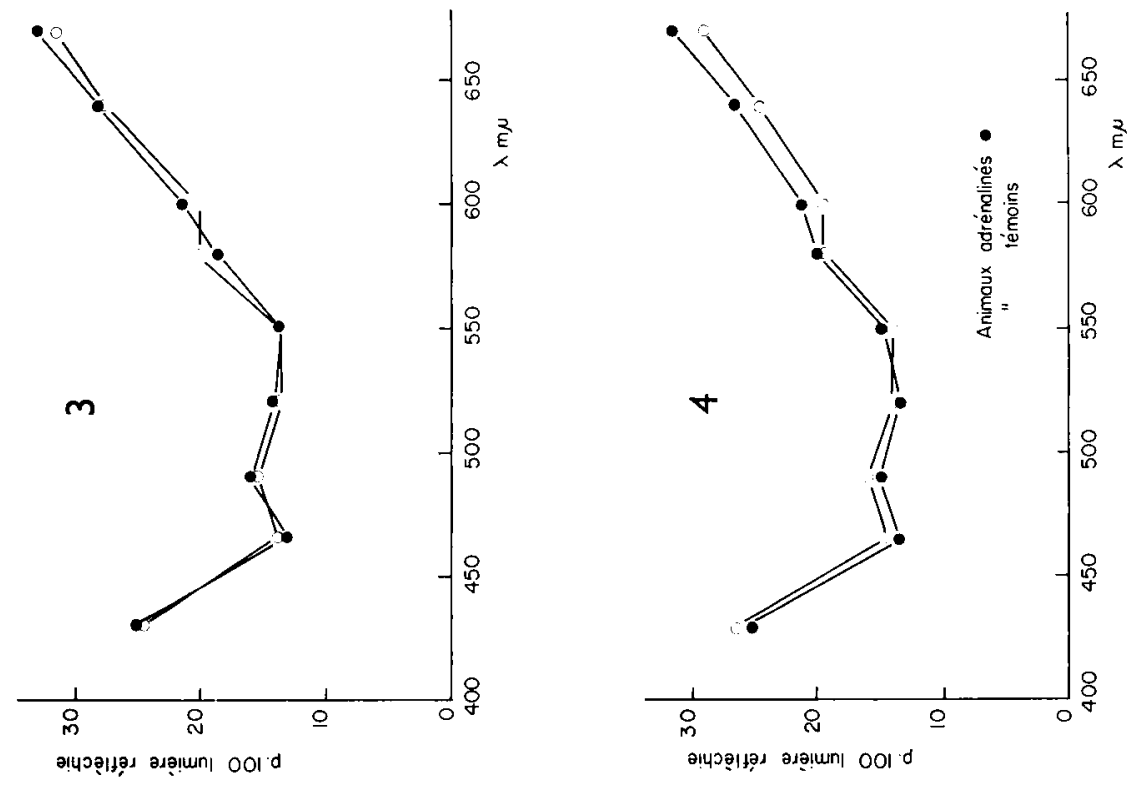

ลิ

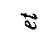

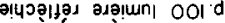
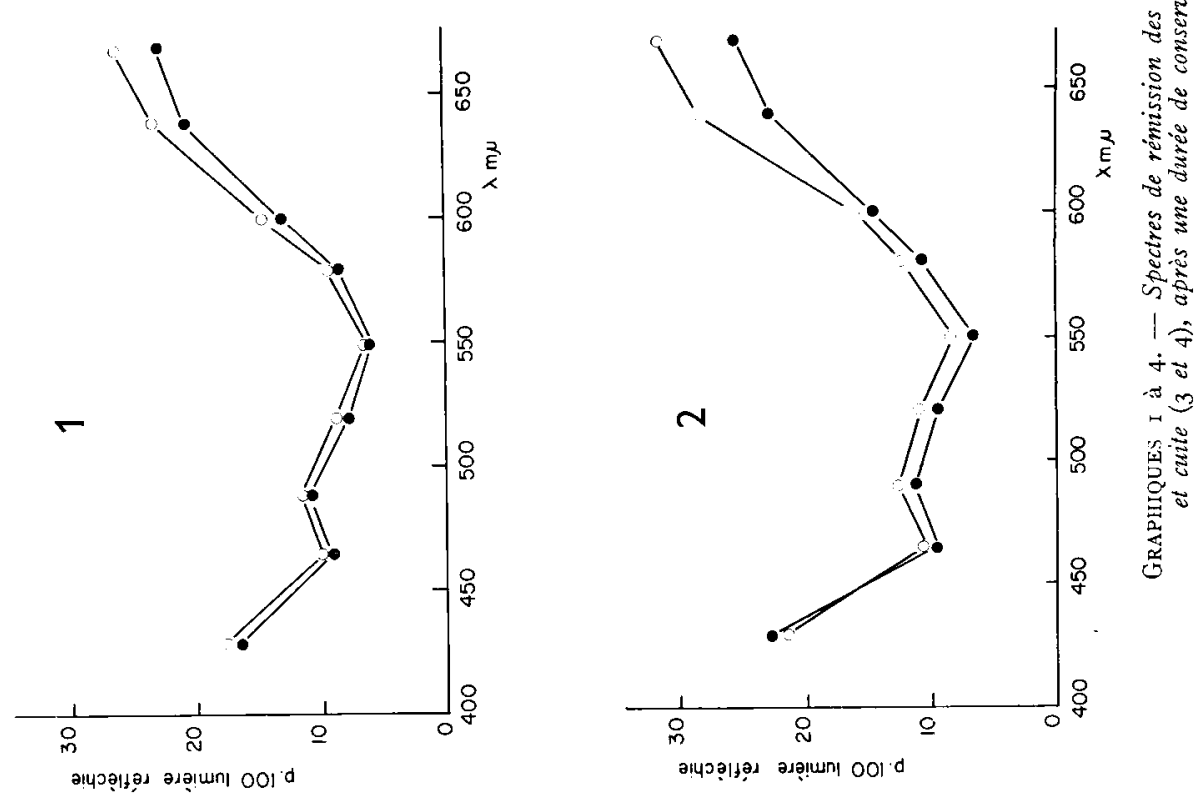
Pendant la conservation $\mathrm{du} 2^{\mathrm{e}}$ au Io $\mathrm{e}^{\mathrm{e}}$ jour, la teneur en pigment des deux groupes reste pratiquement stable, la légère augmentation dans l'un et l'autre groupe pouvant s'expliquer par la perte d'eau lors de la conservation au réfrigérateur.

TABLEAU I

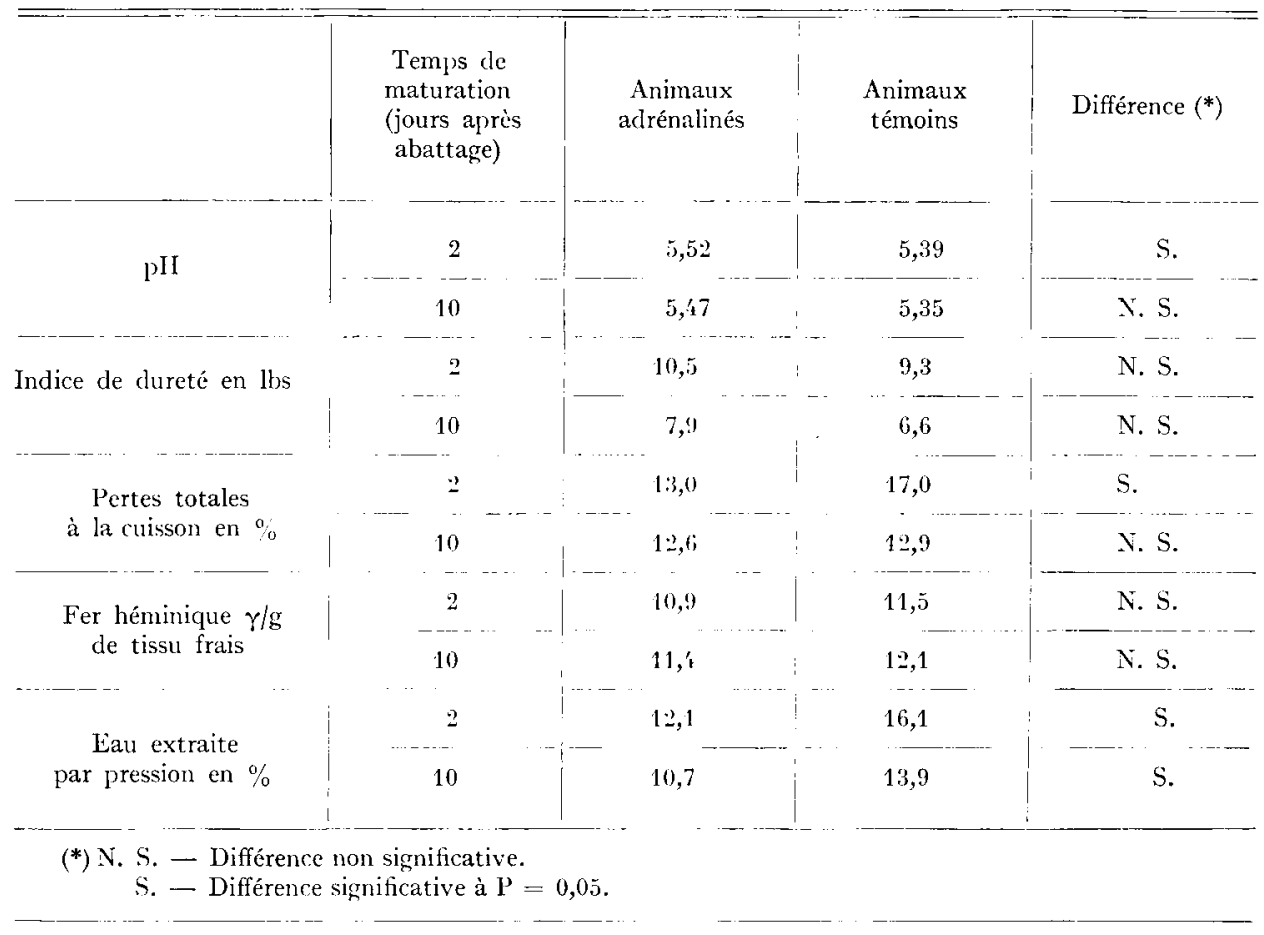

2) Spectre de rémission

a) Muscle cru.

Le graphique I montre que le pourcentage de lumière réfléchie est systématiquement plus faible pour les échantillons provenant des animaux adrénalinés, ce qui confirme l'impression colorée plus foncée que donnent ces muscles à l'examen visuel. Les différences du pourcentage de rémission ne deviennent significatives que dans la zone du spectre visible correspondant aux radiations rouges (filtres 8 et 9) ; toutefois il faut noter que, pour des pourcentages de rémission faibles, une légère diminution de la rémission correspond à une notable différence dans l'impression colorée ressentie.

Étant donné la similitude des teneurs en pigment des muscles des animaux témoins et des animaux adrénalinés, on peut penser que la diminution du pourcentage de rémission est due à une modification de la structure du muscle consécutive à l'injection d'adrénaline.

Le graphique 2 indique que la couleur des muscles des animaux témoins et des animaux adrénalinés a "pâli " pendant la conservation. Cette atténuation 
de la couleur ne peut être imputable à tune éventuelle hétérogénéité du muscle Long dorsal quant à sa teneur en pigment (cf. tabl. I). La baisse de pH constatée au cours de cette même période pourrait expliquer en partie cet éclaircissement. En outre, il est probable que les modifications structurales des protéines musculaires au cours de la maturation agissent sur ce phénomène.

L'éclaircissement est plus important pour les muscles des animaux témoins, ce qui a pour conséquence d'entraîner des différences hautement significatives entre témoins et adrénalinés, après ro jours de conservation.

\section{b) Muscle cuit.}

Les graphiques 3 et 4 indiquent que les différences d'intensité de coloration constatées sur les échantillons frais entre témoins et adrénalinés ne se retrouvent pas après cuisson.

En effet, pour les filtres I à 5, les pourcentages de lumière réfléchie sont très voisins et pour les filtres 6 à 9 , le pourcentage de rémission est même légèrement plus élevé dans le cas des animaux adrénalinés.

L'injection ante mortem d'adrénaline semble donc bien permettre 1'augmentation de la couleur de la viande fraîche. Pour tous les animaux étudiés, nous avons observé chez les animaux traités une coloration plus foncée que chez les témoins, qui est toujours restée dans les limites des colorations jugées acceptables et même souhaitables sur le marché parisien pour la viande de bœuf. Par contre, cette amélioration est réduite à néant par la cuisson. La dénaturation des protéines qu'entraîne cette dernière opération peut expliquer que les légères modifications de structure consécutives à l'injection d'adrénaline ne subsistent pas.

\section{3) $p H$}

Les résultats relatifs au $\mathrm{pH}$ confirment qu'il faut voir dans des modifications de structure du tissu musculaire la voie des modifications de coloration observées. En effet, dans tous les cas, les animaux adrénalinés ont fourni une viande d'un $\mathrm{pH}$ plus élevé que les témoins. Cette différence était, de peu, significative au seuil de 0,05 pour les muscles au frais au jour $\mathrm{J}+2$. L'évolution entre le $2^{\mathrm{e}}$ et le $\mathrm{I}^{\mathrm{e}}$ jour, correspondant à une très légère baisse $\mathrm{du} \mathrm{pH}$, étant sensiblement identique pour les deux groupes, cette différence initiale se retrouvait à la fin des ro jours de conservation (où toutefois elle n'était plus significative).

\section{4) Pouvoir de rétention d'eau}

Les "doubles " des mesures du pouvoir de rétention d'eau par la méthode de pression laissent entrevoir une fidélité insuffisante de cette méthode. Néanmoins, l'étude statistique des résultats montre pour l'ensemble des valeurs obtenues une différence significative entre 1'animal témoin et l'animal adrénaliné pour 5 des 8 couples.

Dans les trois autres, la différence, si elle n'est pas significative, reste dans le même sens.

Il ressort de ces résultats que l'injection d'adrénaline a amélioré le pouvoir de rétention d'eau de la viande (cf. tabl. I). 
Au cours de la conservation, l'évolution du pourcentage d'eau extraite par pression correspond, pour l'un et l'autre groupe, à une diminution que pourraient expliquer les pertes d'eau pendant le stockage au réfrigérateur.

\section{5) Dureté}

La mesure des indices mécaniques de dureté montre une différence en défaveur des animaux adrénalinés (cette différence n'est toutefois pas significative) aussi bien après deux jours qu'après dix jours de conservation.

En ce qui concerne l'évolution pendant la conservation, elle correspond dans l'un et l'autre cas à la poursuite des phénomènes de maturation, entraînant une amélioration de la tendreté (graph. 5).
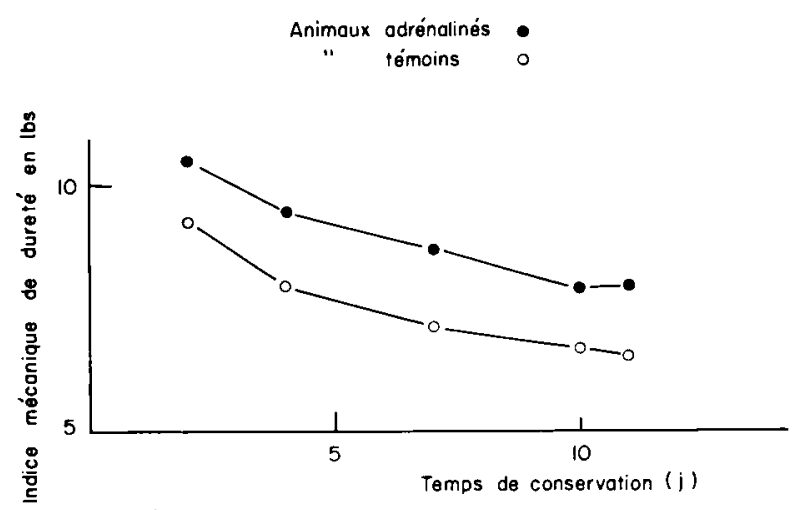

Graphique 5. - Évolution, au cours de la conversation, de la dureté des muscles des animaux témoins et adrénalinés

\section{6) Pertes à la cuisson}

On constate au $2^{\mathbf{e}}$ jour de maturation une différence significative $(\mathrm{P}=0,05)$ entre les deux groupes d'animaux, les animaux témoins ayant un rendement à la cuisson inférieur. Mais cette différence s'atténue rapidement et dès le $4^{\mathrm{e}}$ jour elle a pratiquement disparu. Cette évolution peut, en partie, être due au fait que la viande des animaux adrénalinés a un pouvoir de rétention d'eau supérieur. La perte de poids pendant la conservation au réfrigérateur serait donc plus forte chez les animaux témoins et les pertes à la cuisson de ces mêmes animaux seraient réduites d'autant.

\section{CONCLUSIONS}

L'injection d'adrénaline, à raison de $4 \mathrm{mg}$ par Ioo $\mathrm{kg}$ de poids vif, 20 heures avant l'abattage, a donc permis d'obtenit une viande de coloration plus foncée et plus agréable à l'état cru. Cette amélioration n'est toutefois pas aussi grande que le laissaient prévoir les résultats de HEDRICK (I959), qui considère ces conditions 
d'injection comme optima. Il faut noter que les animaux étudiés appartiennent à des races qu'on doit considérer comme à viande colorée (CHARPENTIER, travaux en cours). L'amélioration serait sans doute plus sensible avec des animaux de races donnant naturellement une viande plus claire.

Mais cette amélioration de la coloration de la viande crue ne se retrouve pas dans la viande cuite ; sans doute les modifications de structure qu'entraîne la cuisson sont-elles très supérieures à celles qu'a provoquées l'injection d'adrénaline.

Il est vraisemblable que seule une augmentation de la teneur en pigment du muscle permettrait une amélioration de la coloration persistant à la cuisson, donc appréciable pour le consommateur.

Les modifications du $\mathrm{pH}$ et du pouvoir de rétention d'eau semblent être plus intéressantes sur le plan pratique, puisqu'elles se traduisent par des améliorations des aptitudes à la conservation des viandes jeunes. L'amélioration du pouvoir de rétention d'eau se traduit également par une diminution des pertes jusqu'au niveau de la cuisson.

Les modifications des caractéristiques de la musculature, dans un sens favorable, qui résultent d'une injection ante mortem d'adrénaline, apparaissent donc en fait plus importantes que ne le laissent penser les degrés de signification des différences statistiques observées.

Reçu pour publication en janvier I964.

\section{SUMMLAR}

POSSIBILITY OF CHANGING SOME PHYSICOCHEMICAL AND TECHNOLOGICAL CHARACTERISTICS OF BEEF MUSCLE BY AN “ ANTE MORTEM " INJECTION OF ADRENALINE

Two groups of eight 16 month old castrated Française Frisonne Pie Noire and Normande animals were used to determine the influence of an ante mortem subcutaneous injection of adrenaline on some physicochemical characteristics of beef muscle. Adrenaline was administrated at the rate of $4 \mathrm{mg}$ per ioo $\mathrm{kg}$ body weight, 20 hours prior to slaughter.

The colour of the meat has been measured by the reflectance method, both on raw and cooked muscle. The darkening observed on raw muscle was no longer noticeable after cooking.

The measurement of the shear-force by the Warner apparatus has shown a slight, non significant increase of toughness in the treated animals.

The most important results was the observed increase of the water holding capacity, as measured by the press-method, and of the cooking yield.

\section{RÉFÉRENCES BIBLIOGRAPHIQUES}

Charpentier J., I963. Résultats non publiés.

de Fremery D., et POOL M. F., I963. The influence of post mortem glycolysis on poultry tenderness.. J. Food Sci., 28, I73-1 76.

Goutefongea R., I 960 . Description d'un nouvel appareil pour mesurer le pouvoir de rétention d'eau de la viande. VIth meeting of Meat Research Institutes. Utrecht.

Hedrick H. B., et al., 1959. Etiology of "dark-cutting" beef. Univ. Missouri. Agr. Exp. St. Res Bull. $\mathrm{n}^{\circ} 7 \mathrm{I} 7$.

Annales de Zootechnie. - 1964 . 
HEDrICK H. B. et al., I96r. Effect of ante mortem administration of adrenaline on post mortem lamb carcass characteristics. J. Anim. Sci., 20, 558-560.

HoRnsey H. C., I956. Colour of cooked cured pork. I. - Estimation of the nitric oxide-haem pigments J. Sci. Food Agric., \%, 534-540.

Vessereau A., r948. Méthodes siatistiques en biologie et en agronomie. Baillière Éd., Paris.

Winkler C. A., I939. Colour of meat. I - Apparatus for its measurement, and relation between pH and colour. Canad.J.Res., 1\%, 1-7. 